\title{
Association between Circulating Fibroblast Growth Factor 23, a-Klotho, and the Left Ventricular Ejection Fraction and Left Ventricular Mass in Cardiology Inpatients
}

\author{
Kensaku Shibata, Shu-ichi Fujita, Hideaki Morita, Yusuke Okamoto, Koichi Sohmiya, Masaaki Hoshiga, \\ Nobukazu Ishizaka*
}

Department of Cardiology, Osaka Medical College, Osaka, Japan

\begin{abstract}
Background: Fibroblast growth factor 23 (FGF23), with its co-receptor Klotho, plays a crucial role in phosphate metabolism. Several recent studies suggested that circulating FGF23 and $\alpha$-Klotho concentrations might be related to cardiovascular abnormalities in patients with advanced renal failure.

Purpose: Using data from 100 cardiology inpatients who were not undergoing chronic hemodialysis, the association of circulating levels of FGF23, $\alpha$-Klotho, and other calcium-phosphate metabolism-related parameters with the left ventricular ejection fraction (LVEF) and left ventricular mass (LVM) was analyzed.

Methods and Results: LVEF was measured using the modified Simpson method for apical 4-chamber LV images and the LVM index (LVMI) was calculated by dividing the LVM by body surface area. Univariate analysis showed that log transformed FGF23, but not that of $\alpha$-Klotho, was significantly associated with LVEF and LVMI with a standardized beta of -0.35 $(P<0.001)$ and $0.26(P<0.05)$, respectively. After adjusting for age, sex, estimated glomerular filtration rate, and serum concentrations of intact parathyroid hormone, and 25-hydroxyvitamin D as covariates into the statistical model, logtransformed FGF23 was found to be a statistically positive predictor for decreased left ventricular function and left ventricular hypertrophy.

Conclusions: In cardiology department inpatients, circulating FGF23 concentrations were found to be associated with the left ventricular mass and LVEF independent of renal function and other calcium-phosphate metabolism-related parameters. Whether modulation of circulating FGF23 levels would improve cardiac outcome in such a high risk population awaits further investigation.
\end{abstract}

Citation: Shibata K, Fujita S-i, Morita H, Okamoto Y, Sohmiya K, et al. (2013) Association between Circulating Fibroblast Growth Factor 23, $\alpha$-Klotho, and the Left Ventricular Ejection Fraction and Left Ventricular Mass in Cardiology Inpatients. PLoS ONE 8(9): e73184. doi:10.1371/journal.pone.0073184

Editor: Rosa Maria Affonso Moysés, Universidade de São Paulo, Brazil

Received April 19, 2013; Accepted July 17, 2013; Published September 9, 2013

Copyright: (c) 2013 Shibata et al. This is an open-access article distributed under the terms of the Creative Commons Attribution License, which permits unrestricted use, distribution, and reproduction in any medium, provided the original author and source are credited.

Funding: The work was supported in part by grants from Meiji Yasuda Life Foundation of Health and Welfare, Mitsui Life Social Welfare Foundation, and Novartis Aging and Geriatric Medicine Research Funding. The funders had no role in study design, data collection and analysis, decision to publish, or preparation of the manuscript.

Competing Interests: Although the work was supported in part by grants from Novartis Aging and Geriatric Medicine Research Funding, there is no relationship with the company relating to employment, consultancy, patents, products in development or marketed products, and other categories that may potently the authors' adherence to all the PLOS ONE policies.

* E-mail: ishizaka@poh.osaka-med.ac.jp

\section{Introduction}

Fibroblast growth factor 23 (FGF23), the most recently identified molecule in the FGF family [1], plays a crucial role in phosphate metabolism, in association with parathyroid hormone (PTH) and vitamin D, by mobilizing sodium-phosphate cotransporters in coordination with Klotho [2,3], a transmembrane protein that has anti-aging properties [4]. Recent studies have shown an association between circulating FGF23 levels and pathologic cardiovascular conditions, including left ventricular hypertrophy [5,6], and vascular endothelial dysfunction [7]. Such associations were investigated mainly in patients with chronic kidney disease $[8,9]$, and in community-dwelling people, albeit in fewer studies [10]. Circulating FGF23 levels are known to be elevated in subjects with severe renal failure, presumably acting against phosphate retention on such occasions [11]. Considering that cardiovascular events are increased in patients with a low estimated glomerular filtration rate (eGFR) [12], the possibility exists that increased FGF23 levels mediate an adverse cardiovascular outcome among patients with end-stage renal disease. Increasingly, clinical and experimental studies have tried to verify this hypothesis. It has been elucidated that Klotho, which is expressed in renal tubular epithelial cells, acts as an obligate coreceptor for FGF23 when FGF23 inhibits proximal tubular phosphate reabsorption leading to a reduction in the serum phosphate level [13]. Although a sensitive and specific assay for 
circulating Klotho ( $\boldsymbol{\alpha}$-Klotho) has become available recently [14], the relationship between Klotho and the cardiovascular system appears to have been less extensively studied; however, the relationship of klotho gene polymorphisms and the serum soluble Klotho $(\alpha$-Klotho) concentration with arteriosclerosis has been suggested in some studies $[9,15]$.

Epidemiological and clinical studies showed that there may be associations between calcium-phosphorus metabolism-related factors other than FGF23/ $\alpha$-Klotho, such as calcium (Ca), inorganic phosphate (IP), PTH, 25-hydroxyvitamin D [25(OH)D], and cardiovascular risk $[16,17,18,19]$. To this end, we have investigated whether circulating levels of FGF23 and $\alpha$-Klotho were associated with the left ventricular ejection fraction (LVEF) and left ventricular hypertrophy independent of these other calciumphosphorus metabolism-related factors in cardiovascular inpatients, a population at high risk for cardiac abnormalities.

\section{Methods}

\section{Ethics Statement}

Written informed consent was obtained from all patients or their guardians. The study was approved by Local Ethics Committee at the Osaka Medical College and conducted in accordance to the Declaration of Helsinki.

\section{Study Population}

The current retrospective study has been approved by the Ethics Committee of Osaka Medical College. Between 2012 January and 2012 December, 102 patients gave informed consent were included in this study in whom sufficient clinical and echocardiographic information, in addition to serum FGF23 and $\alpha$-Klotho concentrations and other calcium-phosphate metabolism-related parameters were available. Two of these patients were found to be undergoing chronic hemodialysis. Because chronic hemodialysis may substantially affect the serum levels of $\alpha$-Klotho and FGF23 [20,21], we excluded these two patients from the study, and thus, total of 100 patients were enrolled in the current study.

\section{Laboratory Analysis}

Blood samples were collected in the morning after an overnight fast. Aliquots of serum and plasma were immediately obtained and stored at -80 degrees until use. Serum level of soluble $\alpha$-Klotho was measured using a solid-phase sandwich enzyme-linked immunosorbent assay (ELISA) (Immuno-Biological Laboratories, Gunma, Japan) [14]. Serum intact FGF23 was measured using a two-step FGF23 enzyme immunoassay (ELISA) kit (Kainos Laboratories Inc., Tokyo, Japan) according to the manufacturer's instructions.

Ca, IP, G-reactive protein (CRP), and B-type natriuretic peptide (BNP) were measured by routine laboratory methods. When serum albumin was $4 \mathrm{mg} / \mathrm{dL}$ or lower, serum Ca levels were corrected using the formula [Ca + (4-"serum albumin")], and designated as corrected Ca (cCa). Serum levels of intact PTH (iPTH), 25(OH)D, and 1,25-dihydroxyvitamin D [1,25( $\left.\mathrm{OH})_{2} \mathrm{D}\right]$ were measured by the immunochemical detection method using electrochemiluminescence, competitive protein binding assay, and radioimmunoassay, respectively (Mitsubishi Medience, Tokyo, Japan). The eGFR was calculated by the following Modification of Diet in Renal Disease equation modified for Japanese subjects: $\mathrm{eGFR}=194 \times(\text { serum creatinine })^{-1.094} \times(\text { age })^{-0.287}(\times 0.739$, when female) [22].

\section{Echocardiography}

Echocardiographic examinations were performed with a Vivid 7 Dimension equipped with a multi-frequency transducer (GE Healthcare, Vingmed, Norway). Left ventricular (LV) end-diastolic dimension (LVDd), interventricular septal thickness (IVST) and posterior wall thickness (PWT) were measured at end diastole. LV volumes were calculated using the modified Simpson method using the apical 4-chamber view. Low LVEF was defined to be present when LVEF was $<50 \%$. For calculation of the LV mass (LVM), we used the formula proposed by Devereux et al. [23] with modification: $0.8 \times 1.04 \times\left[(\mathrm{LVDd}+\mathrm{IVST}+\mathrm{PWT})^{3}-\right.$ $\left.\mathrm{LVDd}^{3}\right]+0.6[24]$. Body surface area (BSA) was calculated using

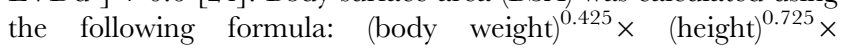
0.007184, and then the LVM index (LVMI) was calculated as the ratio of LVM to the BSA. When the LVMI was greater than 118 (men)/ 108 (women) g/m², LV hypertrophy was defined to be present [25]. Transmitral inflow was recorded using pulsed wave Doppler recordings at the mitral valve leaflet tips in the apical 4chamber view. The peak velocities of early filling $(\mathrm{E})$ and atrial filling (A) were measured. The average of the early peak diastolic mitral annulus velocity obtained at the septal and lateral annulus measured using pulsed wave tissue Doppler imaging, which was designated e'. Because non-sinus cardiac rhythm or certain technical reasons, data on the $\mathrm{E} / \mathrm{A}$ ratio and $\mathrm{E} / \mathrm{e}^{\text {' ratio could be }}$ obtained in 76 and 48 patients, respectively.

\section{Statistical Analysis}

Baseline characteristics were assessed with standard descriptive statistics. Data were expressed either as mean \pm standard deviation, or median and interquartile range (IQR). Spearman rank correlation test was used to assess the correlation between two variables. Univariate or stepwise multivariate linear regression analysis was used to examine the association between LVMI or LVEF and demographic clinical, and laboratory variables. The calciumphosphate metabolism-related parameters, except IP, (FGF23, $\alpha$ Klotho, cCa, intact PTH, 25(OH)D, 1,25( $\left.\mathrm{OH})_{2} \mathrm{D}\right)$ were not found to be normally distributed by the Kolmogorov-Smirnov test; therefore these values, and IP, were log-transformed for multivariate regression analysis although $\alpha$-Klotho and cCa were not found to be completely normalized by $\log$ transformation. Multivariable logistic regression analysis was performed to examine the association between $\log$ (FGF23) concentrations and low LVEF or LV hypertrophy after adjusting for possible confounding variables including eGFR. Data analysis was performed by IBM SPSS statistics version 21.0 (SPSS, Chicago, IL).

\section{Results}

\section{Patient Characteristics}

Demographic and laboratory data and echocardiographic results of the study subjects are shown in Table 1. Among the 100 patients, ischemic heart disease was the most common condition (Table 1). Moderate to severe heart failure (New York Heart Association grades 3 or 4 ) was present in $12 \%$ of the study patients. Neither FGF23 or $\boldsymbol{\alpha}$-Klotho concentrations differed according to the use of angiotensin converting enzyme inhibitor or angiotensin II receptor blocker, beta blocker, calcium channel blocker, or statin (data not shown). Among 100 patients enrolled, 30 patients $(30 \%)$ had eGFR of $\geq 60 \mathrm{~mL} / \mathrm{min} / 1.73 \mathrm{~m}^{2}$ and 70 patients $(70 \%)$ had eGFR of $<60 \mathrm{~mL} / \mathrm{min} / 1.73 \mathrm{~m}^{2}$. Only 7 patients $(7 \%)$ had eGFR of less than $30 \mathrm{~mL} / \mathrm{min} / \mathrm{m}^{2}$. LVDd and LVEF in more than one-fourth of the patients were less than $55 \mathrm{~mm}$ and greater than $50 \%$, respectively, in the current study population (Table 2). FGF23 and $\boldsymbol{\alpha}$-Klotho concentrations were 
Table 1. Demographic characteristics of the study patients.

\begin{tabular}{|c|c|c|c|c|c|c|c|c|c|c|c|c|c|}
\hline \multirow[b]{2}{*}{ Variables } & \multicolumn{3}{|c|}{ Total study patients } & & \multicolumn{3}{|c|}{ eGFR $<60 \mathrm{~mL} / \mathrm{min} / \mathrm{m}^{2}$} & & \multicolumn{4}{|c|}{ eGFR $\geq 60 \mathrm{~mL} / \mathrm{min} / \mathrm{m}^{2}$} & \multirow[b]{2}{*}{ P value } \\
\hline & \multicolumn{4}{|c|}{$(n=100)$} & \multicolumn{4}{|c|}{$(n=70)$} & \multicolumn{4}{|c|}{$(n=30)$} & \\
\hline \multicolumn{14}{|l|}{ Clinical characteristics } \\
\hline Age & 65.4 & \pm & 13.0 & & 68.4 & \pm & 10.9 & & 58.3 & \pm & 14.9 & & $<0.001$ \\
\hline Sex (women/men) & 27 & / & 73 & & 10 & l & 60 & & 17 & I & 13 & & $<0.001$ \\
\hline Body mass index, $\mathrm{kg} / \mathrm{m}^{2}$ & 23.1 & \pm & 3.1 & & 23.2 & \pm & 3.0 & & 23.0 & \pm & 3.2 & & 0.740 \\
\hline Systolic blood pressure, $\mathrm{mmHg}$ & 126.1 & \pm & 19.7 & & 126.5 & \pm & 19.6 & & 125.1 & \pm & 20.1 & & 0.744 \\
\hline Diastolic blood pressure, $\mathrm{mmHg}$ & 71.6 & \pm & 12.4 & & 70.9 & \pm & 12.0 & & 73.3 & \pm & 13.2 & & 0.364 \\
\hline Pulse rate, bpm & 72.3 & \pm & 13.7 & & 72.6 & \pm & 14.9 & & 71.4 & \pm & 10.5 & & 0.695 \\
\hline \multicolumn{14}{|l|}{ Past History } \\
\hline previous $\mathrm{PCl}$ & 44 & ( & 44.0 & ) & 33 & ( & 47.1 & ) & 11 & ( & 36.7 & ) & 0.333 \\
\hline previous CABG & 7 & ( & 7.0 & ) & 4 & ( & 5.7 & ) & 3 & ( & 10.0 & ) & 0.441 \\
\hline \multicolumn{14}{|l|}{ Cardiovascular disease } \\
\hline Ischemic heart disease, $\mathrm{n}(\%)$ & 59 & ( & 59.0 & ) & 43 & ( & 61.4 & ) & 16 & ( & 53.3 & ) & 0.451 \\
\hline Cardiomyopathy, n (\%) & 10 & ( & 10.0 & ) & 7 & ( & 10.0 & ) & 3 & ( & 10.0 & ) & 1.000 \\
\hline NYHA class III/IV, n (\%) & 12 & ( & 12.0 & ) & 5 & ( & 7.1 & ) & 7 & ( & 23.3 & ) & 0.022 \\
\hline Aortic aneurysm, n (\%) & 6 & ( & 6.0 & ) & 5 & ( & 7.1 & ) & 1 & ( & 3.3 & ) & 0.462 \\
\hline Arrythmia, n (\%) & 22 & ( & 22.0 & ) & 12 & ( & 17.1 & ) & 10 & ( & 33.3 & ) & 0.073 \\
\hline Peripheral artery disease, $\mathrm{n}(\%)$ & 8 & ( & 8.0 & ) & 7 & ( & 10.0 & ) & 1 & ( & 3.3 & ) & 0.260 \\
\hline Valvular heart disease, $\mathrm{n}(\%)$ & 8 & ( & 8.0 & ) & 7 & ( & 10.0 & ) & 1 & ( & 3.3 & ) & 0.260 \\
\hline \multicolumn{14}{|l|}{ Cardiac rhythm } \\
\hline Sinus rhythm, n (\%) & 7 & ( & 7.0 & ) & 4 & ( & 5.7 & ) & 3 & ( & 10.0 & ) & 0.687 \\
\hline Atrial fibrillation, n (\%) & 15 & ( & 15.0 & ) & 10 & ( & 14.3 & ) & 5 & ( & 16.7 & ) & \\
\hline Pace maker rhythm, n (\%) & 78 & ( & 78.0 & ) & 56 & ( & 80.0 & ) & 22 & ( & 73.3 & ) & \\
\hline \multicolumn{14}{|l|}{ Smoking status } \\
\hline Never, n (\%) & 33 & ( & 33.0 & ) & 24 & ( & 34.3 & ) & 9 & ( & 30.0 & ) & 0.658 \\
\hline Former, n (\%) & 50 & ( & 50.0 & ) & 33 & ( & 47.1 & ) & 17 & ( & 56.7 & ) & \\
\hline Current, n (\%) & 17 & ( & 17.0 & ) & 13 & ( & 18.6 & ) & 4 & ( & 13.3 & ) & \\
\hline \multicolumn{14}{|l|}{ Medication } \\
\hline ACE inhibitors/ARB, n (\%) & 51 & ( & 51.0 & ) & 41 & ( & 58.6 & ) & 10 & ( & 33.3 & ) & 0.021 \\
\hline Beta blockers, n (\%) & 38 & ( & 38.0 & ) & 32 & ( & 45.7 & ) & 6 & ( & 20.0 & ) & 0.015 \\
\hline Calcium channel blockers, n (\%) & 44 & ( & 44.0 & ) & 35 & ( & 50.0 & ) & 9 & ( & 30.0 & ) & 0.065 \\
\hline Aldosterone antagonist, $\mathrm{n}(\%)$ & 13 & ( & 13.0 & ) & 11 & ( & 15.7 & ) & 2 & ( & 6.7 & ) & 0.218 \\
\hline \multicolumn{14}{|l|}{ Diuretics } \\
\hline Loop, n (\%) & 18 & ( & 18.0 & ) & 15 & ( & 21.4 & ) & 3 & ( & 10.0 & ) & 0.173 \\
\hline Thiazide, n (\%) & 5 & ( & 5.0 & ) & 4 & ( & 5.7 & ) & 1 & ( & 3.3 & ) & 0.617 \\
\hline \multicolumn{14}{|l|}{ Antidiabetic drugs } \\
\hline Sulfonylurea, n (\%) & 10 & ( & 10.0 & ) & 7 & ( & 10.0 & ) & 3 & ( & 10.0 & ) & 1.000 \\
\hline DPP4 inhibitors, n (\%) & 9 & ( & 9.0 & ) & 6 & ( & 8.6 & ) & 3 & ( & 10.0 & ) & 0.819 \\
\hline Insulin, n (\%) & 4 & ( & 4.0 & ) & 2 & ( & 2.9 & ) & 2 & ( & 6.7 & ) & 0.373 \\
\hline Others, n (\%) & 8 & ( & 8.0 & ) & 4 & ( & 5.7 & ) & 4 & ( & 13.3 & ) & 0.198 \\
\hline \multicolumn{14}{|l|}{ Lipid lowering drugs } \\
\hline Statin, n (\%) & 52 & ( & 52.0 & ) & 39 & ( & 55.7 & ) & 13 & ( & 43.3 & ) & 0.256 \\
\hline Fibrate, n (\%) & 1 & ( & 1.0 & ) & 0 & ( & 0.0 & ) & 1 & ( & 3.3 & ) & 0.125 \\
\hline Others, n (\%) & 7 & ( & 7.0 & ) & 6 & ( & 8.6 & ) & 1 & ( & 3.3 & ) & 0.347 \\
\hline
\end{tabular}

$\mathrm{PCl}$, percutaneous coronary intervention; coronary artery bypass graft; ACE, angiotensin converting enzyme; ARB, angiotensin II receptor blocker; DPP4, di-peptidyl peptidase-4. P values are meant to the comparison between eGFR $\geq 60 \mathrm{~mL} / \mathrm{min} / \mathrm{m}^{2}$ and $<60 \mathrm{~mL} / \mathrm{min} / \mathrm{m}^{2}$ groups.

doi:10.1371/journal.pone.0073184.t001 
Table 2. Laboratory and echocardiographic data.

\begin{tabular}{|c|c|c|c|c|c|c|c|c|c|c|c|c|c|c|c|c|c|c|c|}
\hline \multirow[b]{2}{*}{ Variables } & \multicolumn{6}{|c|}{ Total study patients } & \multicolumn{6}{|c|}{ eGFR $<60 \mathrm{~mL} / \mathrm{min} / \mathrm{m}^{2}$} & \multicolumn{6}{|c|}{ eGFR $\geq 60 \mathrm{~mL} / \mathrm{min} / \mathrm{m}^{2}$} & \multirow[b]{2}{*}{$P$ value } \\
\hline & \multicolumn{6}{|c|}{$(n=100)$} & \multicolumn{6}{|c|}{$(n=70)$} & \multicolumn{6}{|c|}{$(n=30)$} & \\
\hline \multicolumn{20}{|l|}{ Laboratory data } \\
\hline White blood cell count, $\times 10^{3} / \mathrm{mL}$ & 5.93 & ( & 4.92 & - & 7.15 & ) & 5.99 & ( & 4.87 & - & 7.25 & ) & 5.86 & ( & 5.07 & - & 6.63 & ) & 0.860 \\
\hline Hemoglobin, g/dL & 13.3 & ( & 12.4 & - & 14.6 & ) & 13.3 & ( & 12.3 & - & 14.5 & ) & 13.7 & ( & 12.4 & - & 14.7 & ) & 0.391 \\
\hline Platelet count, $\times 10^{4} / \mathrm{mL}$ & 21.7 & ( & 18.8 & - & 26.3 & ) & 24.2 & ( & 20.0 & - & 28.4 & ) & 24.2 & ( & 20.0 & - & 28.4 & ) & 0.105 \\
\hline Total protein, $\mathrm{g} / \mathrm{dL}$ & 7.0 & ( & 6.7 & - & 7.4 & ) & 7.0 & ( & 6.8 & - & 7.3 & ) & 6.9 & ( & 6.7 & - & 7.5 & ) & 0.967 \\
\hline Albumin, g/dL & 4.0 & ( & 3.7 & - & 4.2 & ) & 3.9 & ( & 3.7 & - & 4.2 & ) & 4.0 & ( & 3.8 & - & 4.2 & ) & 0.448 \\
\hline Total cholesterol, mg/dL & 178 & ( & 159 & - & 196 & ) & 175 & ( & 155 & - & 196 & ) & 185 & ( & 166 & - & 208 & ) & 0.078 \\
\hline HDL cholesterol, mg/dL & 49 & ( & 42 & - & 63 & ) & 48 & ( & 42 & - & 58 & ) & 54 & ( & 43 & - & 68 & ) & 0.408 \\
\hline LDL cholesterol, mg/dL & 97 & ( & 78 & - & 117 & ) & 95 & ( & 76 & - & 119 & ) & 103 & ( & 84 & - & 116 & ) & 0.065 \\
\hline Triglycerides & 132 & ( & 86 & - & 176 & ) & 126 & ( & 87 & - & 21 & ) & 137 & ( & 82 & - & 189 & ) & 0.321 \\
\hline Blood urea nitrogen & 16.0 & ( & 13.3 & - & 20.0 & ) & 17.0 & ( & 14.0 & - & 21.0 & ) & 14.5 & ( & 11.0 & - & 17.0 & ) & 0.008 \\
\hline Creatinine, $\mathrm{mg} / \mathrm{dL}$ & 0.9 & ( & 0.7 & - & 1.0 & ) & 0.9 & ( & 0.8 & - & 1.1 & ) & 0.7 & ( & 0.6 & - & 0.7 & ) & $<0.001$ \\
\hline eGFR, $\mathrm{mL} / \mathrm{min} / 1.73 \mathrm{~m}^{2}$ & 54 & ( & 42 & - & 64 & ) & 46 & ( & 38 & - & 55 & ) & 69 & ( & 65 & - & 77 & ) & $<0.001$ \\
\hline Alanine transaminase, IU/L & 19 & ( & 14 & - & 27 & ) & 20 & ( & 16 & - & 27 & ) & 20 & ( & 16 & - & 27 & ) & 0.248 \\
\hline Aspartate transaminase, IU/L & 22 & ( & 19 & - & 27 & ) & 21 & ( & 20 & - & 27 & ) & 23 & ( & 18 & - & 27 & ) & 0.851 \\
\hline B-type natriuretic peptide, $\mathrm{pg} / \mathrm{mL}$ & 36.8 & ( & 13.5 & - & 129.0 & ) & 43.1 & ( & 16.1 & - & 176.2 & ) & 29.4 & ( & 9.8 & - & 110.8 & ) & 0.175 \\
\hline C-reactive protein, mg/dL & 0.09 & ( & 0.03 & - & 0.23 & ) & 0.09 & ( & 0.03 & - & 0.20 & ) & 0.09 & ( & 0.05 & - & 0.25 & ) & 0.676 \\
\hline Corrected calcium, mg/dL & 9.2 & ( & 8.9 & - & 9.4 & ) & 9.2 & ( & 8.9 & - & 9.4 & ) & 9.1 & ( & 8.9 & - & 9.4 & ) & 0.561 \\
\hline Inorganic phosphate, mg/dL & 3.4 & ( & 3.0 & - & 3.8 & ) & 3.3 & ( & 3.0 & - & 3.6 & ) & 3.6 & ( & 3.2 & - & 4.0 & ) & 0.019 \\
\hline Intact parathyroid hormone, $\mathrm{pg} / \mathrm{mL}$ & 37.5 & ( & 29.3 & - & 48.8 & ) & 37.5 & ( & 29.8 & - & 25.5 & ) & 37.5 & ( & 25.8 & - & 46.5 & ) & 0.638 \\
\hline 25(OH) Vitamin D, pg/mL & 19.8 & ( & 16.1 & - & 25.0 & ) & 20.7 & ( & 17.4 & - & 25.5 & ) & 16.4 & ( & 13.9 & - & 21.3 & ) & 0.005 \\
\hline $1,25(\mathrm{OH})_{2}$ Vitamin $\mathrm{D}, \mathrm{pg} / \mathrm{mL}$ & 59.0 & ( & 41.0 & - & 80.8 & ) & 59.0 & ( & 41.8 & - & 86.3 & ) & 54.5 & ( & 40.0 & - & 72.5 & ) & 0.359 \\
\hline FGF23, pg/mL & 68.6 & ( & 42.4 & - & 127.0 & ) & 74.5 & ( & 42.5 & - & 151.7 & ) & 64.3 & ( & 41.4 & - & 82.3 & ) & 0.256 \\
\hline$\alpha$-Klotho, pg/mL & 443.3 & ( & 273.7 & - & 595.1 & ) & 443.3 & ( & 268.4 & - & 613.6 & ) & 443.4 & ( & 333.4 & - & 568.6 & ) & 0.729 \\
\hline \multicolumn{20}{|l|}{ Echocardiographic data } \\
\hline LVDd, mm & 4.9 & ( & 4.6 & - & 5.4 & ) & 4.9 & ( & 4.7 & - & 5.4 & ) & 4.8 & ( & 4.4 & - & 5.3 & ) & 0.142 \\
\hline LVDs, mm & 3.2 & ( & 2.8 & - & 3.8 & ) & 3.1 & ( & 2.7 & - & 3.6 & ) & 3.1 & ( & 2.7 & - & 3.6 & ) & 0.362 \\
\hline IVST, mm & 1.0 & ( & 0.9 & - & 1.1 & ) & 1.0 & ( & 0.9 & - & 1.1 & ) & 0.9 & ( & 0.8 & - & 1.0 & ) & 0.052 \\
\hline PWT, mm & 1.0 & ( & 0.9 & - & 1.1 & ) & 1.0 & ( & 0.9 & - & 1.1 & ) & 0.9 & ( & 0.9 & - & 1.0 & ) & 0.156 \\
\hline LVEF, \% & 61 & ( & 53 & - & 65 & ) & 61 & ( & 56 & - & 65 & ) & 62 & ( & 52 & - & 66 & ) & 0.804 \\
\hline LVMI, g/m² & 106 & ( & 86 & - & 127 & ) & 108 & ( & 87 & - & 129 & ) & 96 & ( & 83 & - & 123 & ) & 0.215 \\
\hline$E / A(n=76)$ & 0.8 & ( & 0.6 & - & 1.1 & ) & 0.8 & ( & 0.6 & - & 1.1 & ) & 0.8 & ( & 0.7 & - & 1.1 & ) & 0.562 \\
\hline$E / e^{\prime}(n=48)$ & 8.8 & ( & 7.4 & - & 10.6 & ) & 9.4 & ( & 7.6 & - & 11.9 & ) & 8.2 & ( & 7.1 & - & 9.3 & ) & 0.284 \\
\hline
\end{tabular}

not significantly different between subjects with eGFR of $\geq 60 \mathrm{~mL} / \mathrm{min} / 1.73 \mathrm{~m}^{2}$ and those with eGFR of $<60 \mathrm{~mL} / \mathrm{min} /$ $1.73 \mathrm{~m}^{2}$.

\section{Correlation between $\alpha$-Klotho, FGF23, and Other Clinical and Laboratory Variables}

Correlation between FGF23, $\boldsymbol{\alpha}$-Klotho, and clinical and laboratory parameters were analyzed (Tables 3, 4). FGF23 was found to be correlated negatively with body mass index and hemoglobin concentrations. $\alpha$-Klotho was significantly correlated negatively with blood urea nitrogen and positively with aspartate aminotransferase levels. eGFR showed borderline significant correlation with FGF23, but not with $\alpha$-Klotho. We next investigated the association between log-transformed FGF23 and
$\alpha$-Klotho and other calcium-phosphate metabolism-related parameters (Table 5). It was found that $\log ($ FGF23) was correlated negatively with $\log (\boldsymbol{\alpha}$-Klotho) and positively with $\log (\mathrm{IP})$, and $\log (\boldsymbol{\alpha}$-Klotho $)$ was positively correlated with $\log (25(\mathrm{OH}) \mathrm{D})$ and $\log \left(1,25(\mathrm{OD}){ }_{2} \mathrm{D}\right)$.

\section{Association between calcium-phosphate metabolism- related parameters, LVEF and LVMI}

Univariate linear regression analysis showed that $\log$ (FGF23), $\log (\mathrm{iPTH})$, and $\log (25(\mathrm{OH}) \mathrm{D})$ had significant associations with LVEF and that age, eGFR, $\log ($ FGF23), and $\log (\mathrm{PTH})$ were significant associated with LVMI (Table 6). Standardized Pearson's correlation coefficients of $\log (\mathrm{FGF} 23)$ for LVEF and LVMI were $-0.36(\mathrm{P}=0.003)$ and $0.24(\mathrm{P}=0.045)$, respectively, 
Table 3. Spearman's correlation coefficients between FGF23 and various variables.

\begin{tabular}{|c|c|c|c|c|c|c|}
\hline & \multicolumn{6}{|c|}{ FGF23 } \\
\hline & \multicolumn{2}{|c|}{ Total study patients } & \multicolumn{2}{|c|}{ eGFR $<60 \mathrm{~mL} / \mathrm{min} / \mathrm{m}^{2}$} & \multicolumn{2}{|c|}{ eGFR $\geq 60 \mathrm{~mL} / \mathrm{min} / \mathrm{m}^{2}$} \\
\hline & \multicolumn{2}{|c|}{$(n=100)$} & \multicolumn{2}{|c|}{$(n=70)$} & \multicolumn{2}{|c|}{$(n=30)$} \\
\hline & $\mathbf{r}$ & P value & $\mathbf{r}$ & $P$ value & $\mathbf{r}$ & $P$ value \\
\hline Age, year & -0.03 & \#\#\# & 0.05 & \#\#\# & -0.44 & \#\#\# \\
\hline Sex, $($ male $=1)$ & 0.11 & \#\#\# & -0.01 & \#\#\# & 0.20 & \#\#\# \\
\hline Body mass index & -0.20 & \#\#\# & -0.26 & \#\#\# & -0.13 & $\# \# \#$ \\
\hline Systolic blood pressure & -0.17 & \#\#\# & -0.14 & \#\#\# & -0.26 & \#\#\# \\
\hline Diastolic blood pressure & -0.17 & \#\#\# & -0.16 & \#\#\# & -0.14 & $\# \# \#$ \\
\hline Pulse rate & 0.02 & \#\#\# & 0.18 & \#\#\# & -0.58 & $\# \# \#$ \\
\hline White blood cell count & -0.04 & \#\#\# & -0.14 & \#\#\# & 0.18 & $\# \# \#$ \\
\hline Hemoglobin & -0.24 & \#\#\# & -0.41 & \#\#\# & 0.18 & $\# \# \#$ \\
\hline Platelet count & 0.00 & \#\#\# & 0.04 & \#\#\# & -0.02 & $\# \# \#$ \\
\hline Total protein & 0.13 & \#\#\# & 0.17 & \#\#\# & 0.04 & \#\#\# \\
\hline Albumin & -0.06 & \#\#\# & -0.16 & \#\#\# & 0.19 & \#\#\# \\
\hline Total cholesterol & -0.01 & \#\#\# & -0.04 & \#\#\# & 0.11 & $\# \# \#$ \\
\hline LDL cholesterol & -0.02 & \#\#\# & -0.07 & \#\#\# & 0.12 & $\# \# \#$ \\
\hline HDL cholesterol & 0.06 & \#\#\# & 0.14 & \#\#\# & -0.05 & \#\#\# \\
\hline Triglycerides & -0.05 & \#\#\# & -0.15 & \#\#\# & 0.19 & $\# \# \#$ \\
\hline Blood urea nitrogen & 0.19 & \#\#\# & 0.24 & \#\#\# & -0.01 & \#\#\# \\
\hline Creatinine & 0.20 & \#\#\# & 0.11 & \#\#\# & 0.47 & $\# \# \#$ \\
\hline eGFR & -0.18 & \#\#\# & -0.12 & \#\#\# & -0.29 & $\# \# \#$ \\
\hline Alanine transaminase & -0.13 & \#\#\# & -0.25 & \#\#\# & 0.31 & $\# \# \#$ \\
\hline Aspartate transaminase & -0.14 & \#\#\# & -0.23 & \#\#\# & 0.16 & $\# \# \#$ \\
\hline B-type natriuretic peptide & 0.20 & \#\#\# & 0.16 & \#\#\# & 0.23 & \#\#\# \\
\hline C-reactive protein & 0.03 & \#\#\# & 0.08 & \#\#\# & -0.09 & \#\#\# \\
\hline Corrected calcium & -0.02 & \#\#\# & -0.04 & \#\#\# & 0.03 & \#\#\# \\
\hline Inorganic phosphate & 0.19 & \#\#\# & 0.23 & \#\#\# & 0.18 & \#\#\# \\
\hline Intact parathyroid hormone & 0.07 & \#\#\# & 0.00 & \#\#\# & 0.23 & $\# \# \#$ \\
\hline $25(\mathrm{OH})$ Vitamin $\mathrm{D}, \mathrm{pg} / \mathrm{mL}$ & -0.03 & \#\#\# & -0.07 & \#\#\# & -0.05 & \#\#\# \\
\hline \multirow[t]{5}{*}{$1,25(\mathrm{OH})_{2}$ Vitamin D } & 0.01 & \#\#\# & 0.03 & \#\#\# & -0.06 & \#\#\# \\
\hline & \multicolumn{6}{|c|}{ a-Klotho } \\
\hline & \multicolumn{2}{|c|}{ Total study patients } & \multicolumn{2}{|c|}{ eGFR $<60 \mathrm{~mL} / \mathrm{min} / \mathrm{m}^{2}$} & \multicolumn{2}{|c|}{ eGFR $\geq 60 \mathrm{~mL} / \mathrm{min} / \mathrm{m}^{2}$} \\
\hline & \multicolumn{2}{|c|}{$(n=100)$} & \multicolumn{2}{|c|}{$(n=70)$} & \multicolumn{2}{|c|}{$(n=30)$} \\
\hline & $\mathbf{r}$ & p value & $\mathbf{r}$ & p value & $\mathbf{r}$ & p value \\
\hline Age, year & 0.04 & \#\#\# & 0.07 & \#\#\# & 0.00 & \#\#\# \\
\hline Sex, $($ male $=1)$ & 0.14 & \#\#\# & 0.27 & \#\#\# & 0.00 & $\# \# \#$ \\
\hline Body mass index & 0.08 & \#\#\# & 0.12 & \#\#\# & 0.03 & \#\#\# \\
\hline Systolic blood pressure & 0.16 & \#\#\# & 0.16 & \#\#\# & 0.23 & \#\#\# \\
\hline Diastolic blood pressure & 0.18 & \#\#\# & 0.17 & \#\#\# & 0.27 & \#\#\# \\
\hline Pulse rate & 0.13 & \#\#\# & 0.04 & \#\#\# & 0.41 & \#\#\# \\
\hline White blood cell count & 0.01 & \#\#\# & 0.02 & \#\#\# & -0.09 & \#\#\# \\
\hline Hemoglobin & 0.20 & \#\#\# & 0.24 & \#\#\# & 0.07 & $\# \# \#$ \\
\hline Platelet count & -0.13 & \#\#\# & -0.09 & \#\#\# & -0.28 & \#\#\# \\
\hline Total protein & -0.06 & \#\#\# & -0.11 & \#\#\# & 0.06 & \#\#\# \\
\hline Albumin & 0.19 & \#\#\# & 0.18 & \#\#\# & 0.16 & \#\#\# \\
\hline Total cholesterol & 0.04 & \#\#\# & 0.05 & \#\#\# & -0.03 & \#\#\# \\
\hline LDL cholesterol & 0.02 & \#\#\# & 0.08 & \#\#\# & -0.18 & \#\#\# \\
\hline HDL cholesterol & 0.10 & \#\#\# & 0.05 & \#\#\# & 0.19 & \#\#\# \\
\hline
\end{tabular}


Table 3. Cont.

\begin{tabular}{|c|c|c|c|c|c|c|}
\hline & \multicolumn{6}{|c|}{$\alpha$-Klotho } \\
\hline & \multicolumn{2}{|c|}{ Total study patients } & \multicolumn{2}{|c|}{ eGFR $<60 \mathrm{~mL} / \mathrm{min} / \mathrm{m}^{2}$} & \multicolumn{2}{|c|}{ eGFR $\geq 60 \mathrm{~mL} / \mathrm{min} / \mathrm{m}^{2}$} \\
\hline & \multicolumn{2}{|c|}{$(n=100)$} & \multicolumn{2}{|c|}{$(n=70)$} & \multicolumn{2}{|c|}{$(\mathbf{n}=\mathbf{3 0})$} \\
\hline & $\mathbf{r}$ & $p$ value & $\mathbf{r}$ & $p$ value & $\mathbf{r}$ & $p$ value \\
\hline Triglycerides & -0.05 & \#\#\# & 0.01 & \#\#\# & -0.16 & \#\#\# \\
\hline Blood urea nitrogen & -0.25 & \#\#\# & -0.33 & \#\#\# & -0.08 & \#\#\# \\
\hline Creatinine & -0.15 & \#\#\# & -0.21 & \#\#\# & -0.16 & \#\#\# \\
\hline eGFR & 0.15 & \#\#\# & 0.20 & \#\#\# & 0.24 & \#\#\# \\
\hline Alanine transaminase & 0.15 & \#\#\# & 0.22 & \#\#\# & -0.07 & \#\#\# \\
\hline Aspartate transaminase & 0.23 & \#\#\# & 0.29 & \#\#\# & 0.13 & \#\#\# \\
\hline B-type natriuretic peptide & -0.20 & \#\#\# & -0.24 & \#\#\# & -0.07 & \#\#\# \\
\hline C-reactive protein & -0.16 & \#\#\# & -0.08 & \#\#\# & -0.39 & \#\#\# \\
\hline Corrected calcium & 0.09 & \#\#\# & 0.05 & \#\#\# & 0.13 & \#\#\# \\
\hline Inorganic phosphate & -0.17 & \#\#\# & -0.23 & \#\#\# & -0.10 & \#\#\# \\
\hline Intact parathyroid hormone & -0.08 & \#\#\# & 0.03 & \#\#\# & -0.30 & \#\#\# \\
\hline $25(\mathrm{OH})$ Vitamin $\mathrm{D}, \mathrm{pg} / \mathrm{mL}$ & 0.16 & \#\#\# & 0.10 & \#\#\# & 0.34 & \#\#\# \\
\hline $1,25(\mathrm{OH})_{2}$ Vitamin $\mathrm{D}$ & 0.17 & \#\#\# & 0.17 & \#\#\# & 0.10 & \#\#\# \\
\hline age_2 & -0.03 & \#\#\# & -0.03 & \#\#\# & -0.03 & \#\#\# \\
\hline Sex & 0.11 & \#\#\# & 0.11 & \#\#\# & 0.11 & \#\#\# \\
\hline BMI & -0.20 & \#\#\# & -0.20 & \#\#\# & -0.20 & \#\#\# \\
\hline BPs & -0.17 & \#\#\# & -0.17 & \#\#\# & -0.17 & \#\#\# \\
\hline
\end{tabular}

in 70 patients whose eGFRs were $<60 \mathrm{~mL} / \mathrm{min} / 1.73 \mathrm{~m}^{2}$, and they were $-0.31(\mathrm{P}=0.095)$ and $0.29(\mathrm{P}=0.114)$, respectively, in 30 patients whose eGFRs were $\geq 60 \mathrm{~mL} / \mathrm{min} / 1.73 \mathrm{~m}^{2}$ (Figures 1,2 ). Entering all parameters used in the univariate analysis, stepwise multivariate analysis was performed. Log(FGF23) and lo$\mathrm{g}(25(\mathrm{OH}) \mathrm{D})$ were selected as independent predictors for LVEF, and age, $\log$ (FGF23) and $\log ($ iPTH) were selected as independent predictors for LVMI. Relationship between FGF23, $\alpha$-Klotho, low LVEF, and LV hypertrophy was also investigated by multivariate logistic regression analysis. After adjusting for age, sex, eGFR, $\log (\mathrm{iPTH})$, and $\log (25(\mathrm{OH}) \mathrm{D})$, multivariate logistic regression analysis showed that $\log (\mathrm{FGF} 23)$ showed positive association with low LVEF (significantly) and left ventricular hypertrophy (borderline significantly) with an odds ratio of $13.46 \quad(95 \%$ CI $1.66-109.13, \quad \mathrm{p}=0.015)$ and $2.87 \quad(95 \%$ CI $0.83-9.95$, $\mathrm{p}=0.097)$, respectively.

\section{Discussion}

In the current study, we analyzed the association of circulating levels of FGF23, $\boldsymbol{\alpha}$-Klotho, and other calcium-phosphate metabolism-related parameters with function and mass of the left ventriculum. Univariate analysis showed that $\log$ (FGF23), but not $\log (\alpha$-Klotho), was associated with LVEF and LVMI. It was also found that several other calcium-phosphate metabolism-related parameters; however, after adjusting for such possible confounders, FGF23 remained to be significantly associated negatively with LVEF and positively with LV mass. To the best of our knowledge, this is the first study that targeted cardiology inpatients, namely, a high- risk population for cardiac abnormalities, for the assessment of relationships among FGF23, $\alpha$-Klotho, left ventricular mass, and left ventricular function.
Several previous studies have analyzed the relationship between circulating FGF23/ $\alpha$-Klotho levels in the general population and in subjects with chronic kidney disease (CKD) and more advanced renal failure [26]. By measuring FGF23 levels among 659 community-dwelling women, aged 70-79 years, living in Baltimore, Dalal et al. reported that the median of FGF23 was $34.6 \mathrm{pg} / \mathrm{mL}$ [10], which was lower higher than that in our study. In addition, Westerberg et al. analyzed the FGF23 levels in a population-based cohort of Sweden that included 3014 men aged 69-81 years [27]. The median of FGF23 levels in their study was $43.5 \mathrm{pg} / \mathrm{mL}$, which is slightly lower than that in our study. Ärnlöv et al. reported that higher serum FGF23 was associated with a significantly increased risk for cardiovascular mortality [28] and Mirza et al. reported that FGF23 was positively associated with LV mass in an elderly community population [29]. Of note, several experimental studies have suggested that FGF23 causes pathological hypertrophy of cardiomyocytes [30], which may be mediated by the FGF receptor-dependent activation of the calcineurin-NFAT signaling pathway [6]. The reason why FGF23 levels were found to be slightly higher in our study population remains unclear. On the other hand, it was reported that FGF23 levels was independently associated with LV hypertrophy [6], albeit, the results were not always uniform [31]. Thus, the relatively high FGF23 in our study subjects might be attributed to the relatively high prevalence of LV hypertrophy $(36 \%)$, although ethical and racial differences should be examined in the future.

It has been reported that serum FGF23 levels were significantly elevated in CKD. In the current study, however, FGF23 levels did not significantly differ according to the two eGFR groups. Westerberg et al. reported that, by analyzing the data of adult out patients, FGF23 level was significantly elevated in those with 
Table 4. Spearman's correlation coefficients between $\alpha$-Klotho and various variables.

\begin{tabular}{|c|c|c|c|c|c|c|}
\hline & \multicolumn{6}{|c|}{ FGF23 } \\
\hline & \multicolumn{2}{|c|}{ Total study patients } & \multicolumn{2}{|c|}{ eGFR $<60 \mathrm{~mL} / \mathrm{min} / \mathrm{m}^{2}$} & \multicolumn{2}{|c|}{ eGFR $\geq 60 \mathrm{~mL} / \mathrm{min} / \mathrm{m}^{2}$} \\
\hline & \multicolumn{2}{|c|}{$(n=100)$} & \multicolumn{2}{|c|}{$(n=70)$} & \multicolumn{2}{|c|}{$(\mathbf{n}=\mathbf{3 0})$} \\
\hline & $\mathbf{r}$ & $P$ value & $\mathbf{r}$ & $P$ value & $\mathbf{r}$ & P value \\
\hline Age, year & -0.03 & \#\#\# & 0.05 & \#\#\# & -0.44 & \#\#\# \\
\hline Sex, $($ male $=1)$ & 0.11 & \#\#\# & -0.01 & \#\#\# & 0.20 & \#\#\# \\
\hline Body mass index & -0.20 & \#\#\# & -0.26 & \#\#\# & -0.13 & \#\#\# \\
\hline Systolic blood pressure & -0.17 & \#\#\# & -0.14 & \#\#\# & -0.26 & \#\#\# \\
\hline Diastolic blood pressure & -0.17 & \#\#\# & -0.16 & \#\#\# & -0.14 & \#\#\# \\
\hline Pulse rate & 0.02 & \#\#\# & 0.18 & \#\#\# & -0.58 & \#\#\# \\
\hline White blood cell count & -0.04 & \#\#\# & -0.14 & \#\#\# & 0.18 & \#\#\# \\
\hline Hemoglobin & -0.24 & \#\#\# & -0.41 & \#\#\# & 0.18 & \#\#\# \\
\hline Platelet count & 0.00 & \#\#\# & 0.04 & \#\#\# & -0.02 & \#\#\# \\
\hline Total protein & 0.13 & \#\#\# & 0.17 & \#\#\# & 0.04 & \#\#\# \\
\hline Albumin & -0.06 & \#\#\# & -0.16 & \#\#\# & 0.19 & \#\#\# \\
\hline Total cholesterol & -0.01 & \#\#\# & -0.04 & \#\#\# & 0.11 & \#\#\# \\
\hline LDL cholesterol & -0.02 & \#\#\# & -0.07 & \#\#\# & 0.12 & \#\#\# \\
\hline HDL cholesterol & 0.06 & \#\#\# & 0.14 & \#\#\# & -0.05 & \#\#\# \\
\hline Triglycerides & -0.05 & \#\#\# & -0.15 & \#\#\# & 0.19 & \#\#\# \\
\hline Blood urea nitrogen & 0.19 & \#\#\# & 0.24 & \#\#\# & -0.01 & \#\#\# \\
\hline Creatinine & 0.20 & \#\#\# & 0.11 & \#\#\# & 0.47 & \#\#\# \\
\hline eGFR & -0.18 & \#\#\# & -0.12 & \#\#\# & -0.29 & \#\#\# \\
\hline Alanine transaminase & -0.13 & \#\#\# & -0.25 & \#\#\# & 0.31 & \#\#\# \\
\hline Aspartate transaminase & -0.14 & \#\#\# & -0.23 & \#\#\# & 0.16 & \#\#\# \\
\hline B-type natriuretic peptide & 0.20 & \#\#\# & 0.16 & \#\#\# & 0.23 & \#\#\# \\
\hline C-reactive protein & 0.03 & \#\#\# & 0.08 & \#\#\# & -0.09 & \#\#\# \\
\hline Corrected calcium & -0.02 & \#\#\# & -0.04 & \#\#\# & 0.03 & \#\#\# \\
\hline Inorganic phosphate & 0.19 & \#\#\# & 0.23 & \#\#\# & 0.18 & \#\#\# \\
\hline Intact parathyroid hormone & 0.07 & \#\#\# & 0.00 & \#\#\# & 0.23 & \#\#\# \\
\hline $25(\mathrm{OH})$ Vitamin $\mathrm{D}, \mathrm{pg} / \mathrm{mL}$ & -0.03 & \#\#\# & -0.07 & \#\#\# & -0.05 & \#\#\# \\
\hline \multirow[t]{5}{*}{$1,25(\mathrm{OH})_{2}$ Vitamin D } & 0.01 & \#\#\# & 0.03 & \#\#\# & -0.06 & \#\#\# \\
\hline & \multicolumn{6}{|c|}{$\alpha-K l o t h o$} \\
\hline & \multicolumn{2}{|c|}{ Total study patients } & \multicolumn{2}{|c|}{ eGFR $<60 \mathrm{~mL} / \mathrm{min} / \mathrm{m}^{2}$} & \multicolumn{2}{|c|}{ eGFR $\geq 60 \mathrm{~mL} / \mathrm{min} / \mathrm{m}^{2}$} \\
\hline & \multicolumn{2}{|c|}{$(n=100)$} & \multicolumn{2}{|c|}{$(n=70)$} & \multicolumn{2}{|c|}{$(n=30)$} \\
\hline & $\mathbf{r}$ & p value & $\mathbf{r}$ & p value & $\mathbf{r}$ & p value \\
\hline Age, year & 0.04 & \#\#\# & 0.07 & \#\#\# & 0.00 & \#\#\# \\
\hline Sex, $($ male $=1)$ & 0.14 & \#\#\# & 0.27 & \#\#\# & 0.00 & \#\#\# \\
\hline Body mass index & 0.08 & \#\#\# & 0.12 & \#\#\# & 0.03 & \#\#\# \\
\hline Systolic blood pressure & 0.16 & \#\#\# & 0.16 & \#\#\# & 0.23 & \#\#\# \\
\hline Diastolic blood pressure & 0.18 & \#\#\# & 0.17 & \#\#\# & 0.27 & \#\#\# \\
\hline Pulse rate & 0.13 & \#\#\# & 0.04 & \#\#\# & 0.41 & \#\#\# \\
\hline White blood cell count & 0.01 & \#\#\# & 0.02 & \#\#\# & -0.09 & \#\#\# \\
\hline Hemoglobin & 0.20 & \#\#\# & 0.24 & \#\#\# & 0.07 & \#\#\# \\
\hline Platelet count & -0.13 & \#\#\# & -0.09 & \#\#\# & -0.28 & \#\#\# \\
\hline Total protein & -0.06 & \#\#\# & -0.11 & \#\#\# & 0.06 & \#\#\# \\
\hline Albumin & 0.19 & \#\#\# & 0.18 & \#\#\# & 0.16 & \#\#\# \\
\hline Total cholesterol & 0.04 & \#\#\# & 0.05 & \#\#\# & -0.03 & \#\#\# \\
\hline LDL cholesterol & 0.02 & \#\#\# & 0.08 & \#\#\# & -0.18 & \#\#\# \\
\hline HDL cholesterol & 0.10 & \#\#\# & 0.05 & \#\#\# & 0.19 & \#\#\# \\
\hline
\end{tabular}


Table 4. Cont.

\begin{tabular}{|c|c|c|c|c|c|c|}
\hline & \multicolumn{6}{|c|}{ a-Klotho } \\
\hline & \multicolumn{2}{|c|}{ Total study patients } & \multicolumn{2}{|c|}{ eGFR $<60 \mathrm{~mL} / \mathrm{min} / \mathrm{m}^{2}$} & \multicolumn{2}{|c|}{ eGFR $\geq 60 \mathrm{~mL} / \mathrm{min} / \mathrm{m}^{2}$} \\
\hline & \multicolumn{2}{|c|}{$(n=100)$} & \multicolumn{2}{|c|}{$(n=70)$} & \multicolumn{2}{|c|}{$(\mathbf{n}=\mathbf{3 0})$} \\
\hline & $\mathbf{r}$ & $p$ value & $\mathbf{r}$ & $p$ value & $\mathbf{r}$ & $p$ value \\
\hline Triglycerides & -0.05 & \#\#\# & 0.01 & \#\#\# & -0.16 & \#\#\# \\
\hline Blood urea nitrogen & -0.25 & \#\#\# & -0.33 & \#\#\# & -0.08 & \#\#\# \\
\hline Creatinine & -0.15 & \#\#\# & -0.21 & \#\#\# & -0.16 & \#\#\# \\
\hline eGFR & 0.15 & \#\#\# & 0.20 & \#\#\# & 0.24 & \#\#\# \\
\hline Alanine transaminase & 0.15 & \#\#\# & 0.22 & \#\#\# & -0.07 & \#\#\# \\
\hline Aspartate transaminase & 0.23 & \#\#\# & 0.29 & \#\#\# & 0.13 & \#\#\# \\
\hline B-type natriuretic peptide & -0.20 & \#\#\# & -0.24 & \#\#\# & -0.07 & \#\#\# \\
\hline C-reactive protein & -0.16 & \#\#\# & -0.08 & \#\#\# & -0.39 & \#\#\# \\
\hline Corrected calcium & 0.09 & \#\#\# & 0.05 & \#\#\# & 0.13 & \#\#\# \\
\hline Inorganic phosphate & -0.17 & \#\#\# & -0.23 & \#\#\# & -0.10 & \#\#\# \\
\hline Intact parathyroid hormone & -0.08 & \#\#\# & 0.03 & \#\#\# & -0.30 & \#\#\# \\
\hline 25(OH) Vitamin $\mathrm{D}, \mathrm{pg} / \mathrm{mL}$ & 0.16 & \#\#\# & 0.10 & \#\#\# & 0.34 & \#\#\# \\
\hline $1,25(\mathrm{OH})_{2}$ Vitamin D & 0.17 & \#\#\# & 0.17 & \#\#\# & 0.10 & \#\#\# \\
\hline age_2 & -0.03 & \#\#\# & -0.03 & \#\#\# & -0.03 & \#\#\# \\
\hline Sex & 0.11 & \#\#\# & 0.11 & \#\#\# & 0.11 & \#\#\# \\
\hline BMI & -0.20 & \#\#\# & -0.20 & \#\#\# & -0.20 & \#\#\# \\
\hline BPs & -0.17 & \#\#\# & -0.17 & \#\#\# & -0.17 & \#\#\# \\
\hline
\end{tabular}

CKD stage 4 or 5, but not in those with CKD stage 3, compared with subjects with CKD stage 1-2 [32]. We excluded patients undergoing chronic hemodialysis from the study population, and only 7 patients in the current study had eGFR of less than $30 \mathrm{~mL} /$ $\mathrm{min} / 1.73 \mathrm{~m}^{2}$, which may, at least in part, explain why FGF23 level did not significantly differ between low eGFR $(<60 \mathrm{~mL} /$ $\left.\mathrm{min} / \mathrm{m}^{2}\right)$ and preserved eGFR $\left(\geq 60 \mathrm{~mL} / \mathrm{min} / \mathrm{m}^{2}\right)$ groups. We found that correlation between $\log$ (FGF23) and LVEF was (borderline) significant in patients with eGFRs of $\geq 60 \mathrm{~mL} / \mathrm{min}$ / $1.73 \mathrm{~m}^{2}$. It may suggest the possibility that FGF23 plays a role in the cardiac remodeling even in non-CKD subjects or that FGF23 is regulated by certain factors that are modulated in response to cardiac performance. Considering that FGF23 has now been considered to mediate 'off-target,' direct, end-organ toxicity in the heart [33], the strength of relationship between FGF23 and cardiac parameters in various CKD stages should be investigated in more detail in future studies.

The relationship between circulating $\alpha$-Klotho in the general population and in patients with cardiovascular risk seems to have been less extensively assessed.

Kim et al. analyzed the $\alpha$-Klotho level in patients with various renal functions among the GKD cohort [34]. They found that $\alpha$ Klotho level showed graded decrease according to the CKD stage; the median of $\alpha$-Klotho were $540 \mathrm{pg} / \mathrm{mL}$ in patients with CKD stage 1 and $296 \mathrm{pg} / \mathrm{m}$ in those with CKD stage 5 . In addition, Kacso et al. reported that the median of $\alpha$-Klotho concentrations was $400 \mathrm{pg} / \mathrm{mL}$ in diabetic patients without CKD and $800 \mathrm{pg} /$ $\mathrm{mL}$ in controls [35]. The median of the serum $\alpha$-Klotho

Table 5. Pearson's correlation coefficients between variable related to calcium-phosphate metabolism.

\begin{tabular}{|c|c|c|c|c|c|c|c|c|c|c|c|c|c|c|}
\hline & \multicolumn{2}{|c|}{$\log (F G F 23)$} & \multicolumn{2}{|c|}{$\log (\alpha-K l o t h o)$} & \multicolumn{2}{|c|}{$\log (c C a)$} & \multicolumn{2}{|c|}{$\log (I P)$} & \multicolumn{2}{|c|}{$\log (\mathrm{iPTH})$} & \multicolumn{2}{|c|}{$\begin{array}{l}\log (25 \\
(\mathrm{OH}) \mathrm{D})\end{array}$} & \multicolumn{2}{|c|}{$\begin{array}{l}\log (1,25 \\
\left.(\mathrm{OH})_{2} \mathrm{D}\right)\end{array}$} \\
\hline & $\mathbf{r}$ & p value & $\mathbf{r}$ & p value & $\mathbf{r}$ & p value & $\mathbf{r}$ & p value & $\mathbf{r}$ & p value & $\mathbf{r}$ & p value & $\mathbf{r}$ & p value \\
\hline $\log (F G F 23)$ & - & & -0.25 & 0.013 & -0.02 & 0.874 & 0.19 & 0.056 & 0.07 & 0.503 & -0.03 & 0.759 & 0.01 & 0.917 \\
\hline $\log (\alpha-K$ lotho $)$ & -0.25 & 0.013 & - & & 0.09 & 0.390 & -0.17 & 0.091 & -0.08 & 0.428 & 0.16 & 0.114 & 0.17 & 0.083 \\
\hline $\log (\mathrm{cCa})$ & -0.02 & 0.874 & 0.09 & 0.390 & - & & 0.12 & 0.231 & 0.06 & 0.555 & 0.20 & 0.042 & 0.02 & 0.822 \\
\hline $\log (\mathrm{IP})$ & 0.19 & 0.056 & -0.17 & 0.091 & 0.12 & 0.231 & - & & 0.05 & 0.642 & -0.04 & 0.684 & -0.08 & 0.446 \\
\hline Log(iPTH) & 0.07 & 0.503 & -0.08 & 0.428 & 0.06 & 0.555 & 0.05 & 0.642 & - & & -0.09 & 0.359 & -0.12 & 0.233 \\
\hline $\log (25(\mathrm{OH}) \mathrm{D})$ & -0.03 & 0.759 & 0.16 & 0.114 & 0.21 & 0.040 & -0.04 & 0.716 & -0.10 & 0.325 & - & & 0.30 & 0.003 \\
\hline $\log \left(1,25(\mathrm{OH})_{2} \mathrm{D}\right)$ & 0.01 & 0.917 & 0.17 & 0.083 & 0.02 & 0.822 & -0.08 & 0.446 & -0.12 & 0.233 & 0.30 & 0.002 & - & \\
\hline
\end{tabular}



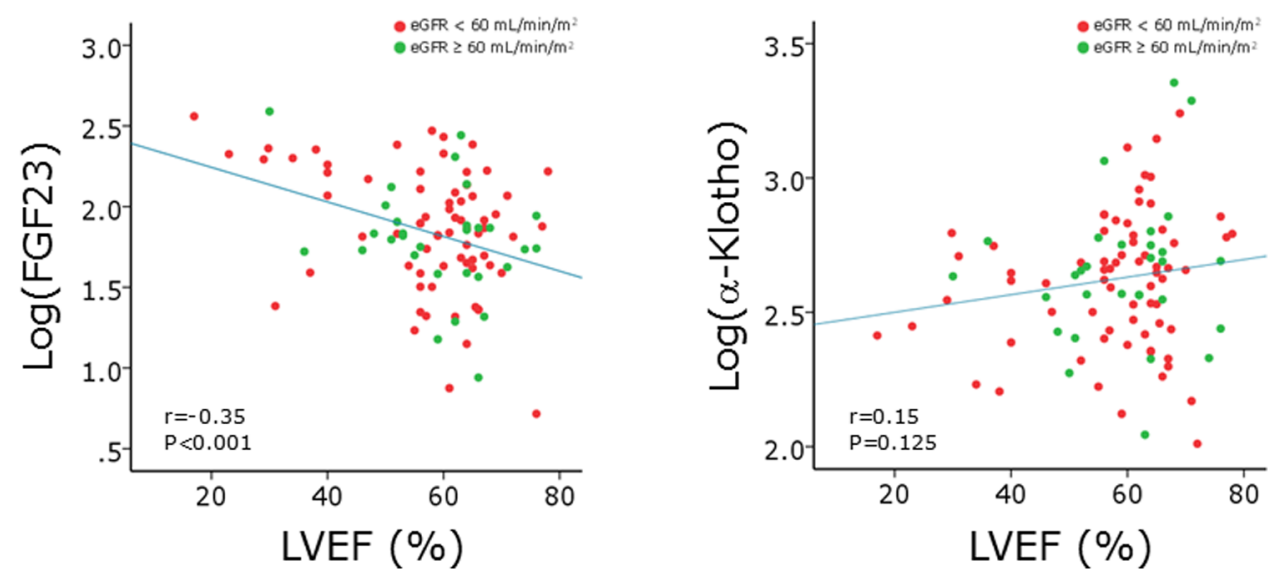

Figure 1. Correlation between FGF23, $\alpha$-Klotho and left ventricular ejection fraction (LVEF). A. Correlation between log(FGF23) and LVEF. B. Correlation between log $(\alpha$-Klotho) and LVEF. Pearson's correlation analysis was performed. Green and red closed circles indicate patients with eGFR of $\geq 60 \mathrm{~mL} / \mathrm{min} / 1.73 \mathrm{~m}^{2}$ and those with eGFR of $<60 \mathrm{~mL} / \mathrm{min} / 1.73 \mathrm{~m}^{2}$, respectively. doi:10.1371/journal.pone.0073184.g001

concentrations, that was $504 \mathrm{pg} / \mathrm{mL}$, may be said to be within a similar range of these previous studies. Semba et al. reported that higher plasma Klotho concentrations are independently associated with a lower likelihood of having cardiovascular disease in community-dwelling adults [36]. In the current study, unlike FGF23, Klotho was not associated with LVMI or LVEF, which might account for lack of reports on this topic. It should be noted, however, a recent animal experiment showed that Klotho may reduce TRPC6 channels present at the cellular surface leading to the reduction of the inward calcium current under certain condition of stress, resulting in protecting the heart from hypertrophy and systolic dysfunction [37].

In the current study, multivariate analysis showed that 25(OH)D and iPTH were positively associated with LVEF and LVMI, respectively, findings that were in accordance with previous observations [38,39], although such associations may depend on the target population [40]. Considering that LV hypertrophy and reduced LVEF are parameters predictive of poor cardiovascular outcome [41], and that both circulating levels of FGF23 and $\alpha$-Klotho are regulated in patients with chronic kidney disease or advanced renal failure [42], alterations in the circulating levels or activity of FGF23/ $\alpha$-Klotho may link the chronic kidney disease with higher cardiovascular risk [43]. Circulating levels of $\mathrm{Ca}$, IP, and $25(\mathrm{OH}) \mathrm{D}$ were also reported to be associated with cardiovascular risk [44,45] and elevated PTH levels were associated with left ventricular mass and severity of heart failure $[39,46]$; therefore, one should confirm, as has been done in the current study, whether the observed relationship between FGF23/ $\alpha$-Klotho and cardiac abnormality is mediated or confounded by the above-mentioned variables, whose levels are related to FGF23/ $\alpha$-Klotho levels $[47,48]$.

There are several limitations in the current study. First, the current study included patients with various cardiovascular diseases. The relationship between FGF23 and cardiac remodeling
A

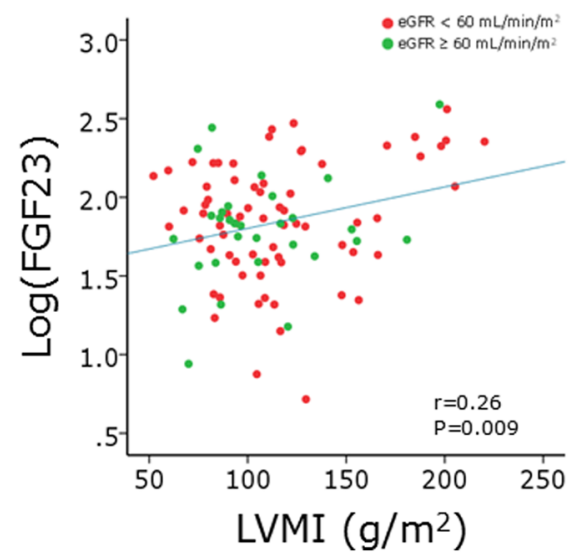

B

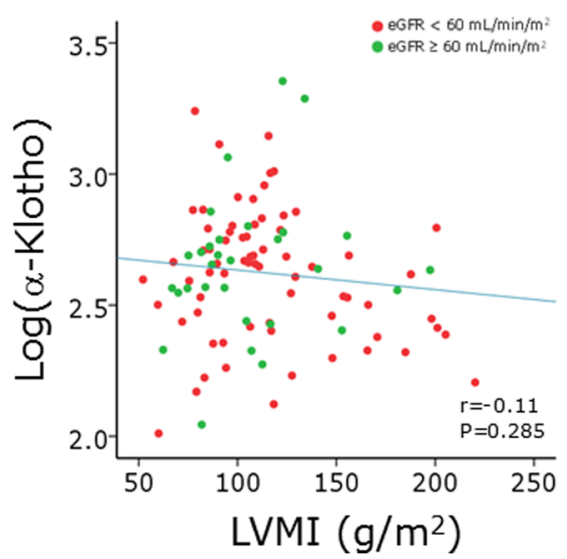

Figure 2. Correlation between FGF23, $\alpha$-Klotho and left ventricular mass index (LVMI). A. Correlation between log(FGF23) and LVMI. B. Correlation between $\log (\alpha-K l o t h o)$ and LVMI. Pearson's correlation analysis was performed. Green and red closed circles indicate patients with eGFR of $\geq 60 \mathrm{~mL} / \mathrm{min} / 1.73 \mathrm{~m}^{2}$ and those with eGFR of $<60 \mathrm{~mL} / \mathrm{min} / 1.73 \mathrm{~m}^{2}$, respectively.

doi:10.1371/journal.pone.0073184.g002 
Table 6. Univariate and multivariate analysis for the association with ejection fraction and mass index of the left ventriculum.

\begin{tabular}{|c|c|c|c|c|c|c|c|c|c|c|c|c|c|c|c|}
\hline \multicolumn{8}{|c|}{ Univariate analysis } & \multicolumn{8}{|c|}{ Multivariate analysis } \\
\hline \multirow[b]{2}{*}{ Predictors } & \multicolumn{7}{|l|}{ Standardized } & & \multicolumn{7}{|l|}{ Standardized } \\
\hline & $\begin{array}{l}\text { correlation } \\
\text { coefficient }\end{array}$ & & \multicolumn{4}{|c|}{$(95 \% \mathrm{Cl})$} & P value & $\begin{array}{l}\text { Selected } \\
\text { predictors }\end{array}$ & $\begin{array}{l}\text { correlation } \\
\text { coefficient }\end{array}$ & & \multicolumn{4}{|c|}{$(95 \% \mathrm{Cl})$} & $P$ value \\
\hline \multicolumn{16}{|c|}{ Dependent variable: LVEF } \\
\hline Age & -0.01 & ( & -0.21 & - & 0.19 & ) & 0.943 & & & & & & & & \\
\hline Sex $($ male $=1)$ & -0.16 & ( & -0.35 & - & 0.04 & ) & 0.124 & & & & & & & & \\
\hline Systolic BP & 0.07 & ( & -0.13 & - & 0.27 & ) & 0.490 & & & & & & & & \\
\hline eGFR & 0.16 & ( & -0.04 & - & 0.36 & ) & 0.112 & & & & & & & & \\
\hline $\log ($ FGF23) & -0.35 & ( & -0.54 & - & -0.16 & ) & $<0.001$ & $\log (\mathrm{FGF} 23)$ & -0.34 & ( & -0.52 & & -0.16 & & $<0.001$ \\
\hline $\log (\alpha-$ Klotho $)$ & 0.15 & ( & -0.04 & - & 0.35 & ) & 0.125 & & & & & & & & \\
\hline $\log (\mathrm{cCa})$ & -0.06 & ( & -0.26 & - & 0.14 & ) & 0.524 & & & & & & & & \\
\hline $\log (I P)$ & 0.04 & ( & -0.16 & - & 0.24 & ) & 0.665 & & & & & & & & \\
\hline $\log (\mathrm{iPTH})$ & -0.23 & ( & -0.42 & - & -0.03 & ) & 0.022 & & & & & & & & \\
\hline $\log (25(\mathrm{OH}) \mathrm{D})$ & 0.24 & ( & 0.04 & - & 0.43 & ) & 0.018 & $\log (25(\mathrm{OH}) \mathrm{D})$ & 0.22 & ( & 0.04 & - & 0.41 & ) & 0.018 \\
\hline $\log \left(1,25(\mathrm{OH})_{2} \mathrm{D}\right)$ & 0.17 & ( & -0.02 & - & 0.37 & ) & 0.083 & & & & & & & & \\
\hline \multicolumn{16}{|c|}{ Dependent variable: LVMI } \\
\hline Age & 0.28 & ( & 0.08 & - & 0.47 & ) & 0.005 & Age & 0.29 & ( & 0.10 & - & 0.47 & ) & 0.002 \\
\hline Sex $($ male $=1)$ & 0.02 & ( & -0.18 & - & 0.22 & ) & 0.829 & & & & & & & & \\
\hline Systolic BP & 0.14 & ( & -0.06 & - & 0.34 & ) & 0.172 & & & & & & & & \\
\hline eGFR & -0.21 & ( & -0.41 & - & -0.02 & ) & 0.034 & & & & & & & & \\
\hline $\log (F G F 23)$ & 0.26 & ( & 0.07 & - & 0.45 & ) & 0.009 & $\log (\mathrm{FGF} 23)$ & 0.25 & ( & 0.07 & - & 0.43 & ) & 0.007 \\
\hline $\log (\alpha-$ Klotho $)$ & -0.11 & ( & -0.31 & - & 0.09 & 1 & 0.285 & & & & & & & & \\
\hline $\log (c \mathrm{Ca})$ & 0.12 & ( & -0.08 & - & 0.32 & ) & 0.229 & & & & & & & & \\
\hline $\log (\mathbb{I P})$ & -0.03 & ( & -0.23 & - & 0.17 & ) & 0.801 & & & & & & & & \\
\hline $\log (\mathrm{iPTH})$ & 0.25 & ( & 0.05 & - & 0.44 & ) & 0.013 & $\log (\mathrm{iPTH})$ & 0.22 & ( & 0.04 & - & 0.40 & ) & 0.018 \\
\hline $\log (25(\mathrm{OH}) \mathrm{D})$ & 0.05 & ( & -0.16 & - & 0.25 & ) & 0.655 & & & & & & & & \\
\hline $\log \left(1,25(\mathrm{OH})_{2} \mathrm{D}\right)$ & -0.01 & ( & -0.21 & - & 0.19 & ) & 0.903 & & & & & & & & \\
\hline
\end{tabular}

LVEF: left ventricular ejection fraction; LVMI; left ventricular mass index, BP; blood pressure. For multivariate analysis, stepwise regression analysis was performed by entering all the variables listed for the univariate analysis. Parameters that remained to be significant predictors are listed.

doi:10.1371/journal.pone.0073184.t006

might differ according the etiology of the background disorders. Second, although exposure of primary cardiomyocytes to FGF23 was reported to result in elevated intracellular calcium and increased ventricular muscle strip contractility in the experimental model [30], whether FGF23 truly represents a useful biomarker, or is a simple bystander, for cardiac dysfunction and hypertrophy should be analyzed in the longitudinal studies. In addition, whether life-style modifications or drug interventions that might reduce circulating FGF23 levels would improve the cardiovascular prognosis should be investigated.

In summary, by analyzing data from cardiology inpatients, we found that circulating levels of FGF23 were positively associated with LV mass, LV hypertrophy, reduced LV systolic function, and plasma BNP concentration. This relationship was statistically

\section{References}

1. Yamashita T, Yoshioka M, Itoh N (2000) Identification of a novel fibroblast growth factor, FGF-23, preferentially expressed in the ventrolateral thalamic nucleus of the brain. Biochem Biophys Res Commun 277: 494-498.

2. Kuro-o M, Matsumura Y, Aizawa H, Kawaguchi H, Suga T, et al. (1997) Mutation of the mouse klotho gene leads to a syndrome resembling ageing. Nature 390: 45-51.

3. Kurosu H, Yamamoto M, Clark JD, Pastor JV, Nandi A, et al. (2005) Suppression of aging in mice by the hormone Klotho. Science 309: 1829-1833. significant after adjustment for other calcium-phosphate metabolism-related parameters including $\alpha$-Klotho. Whether modulation of FGF23 activity would improve cardiac outcome in such a highrisk population awaits further investigation.

\section{Acknowledgments}

We are highly appreciative of Chieko Ohta, Yumiko Ohgami, and Megumi Hashimoto for their excellent technical assistance.

\section{Author Contributions}

Conceived and designed the experiments: NI. Analyzed the data: K. Sohmiya SF HM NI. Contributed reagents/materials/analysis tools: K. Shibata MH YO. Wrote the paper: K. Shibata NI.

4. Krajisnik T, Bjorklund P, Marsell R, Ljunggren O, Akerstrom G, et al. (2007) Fibroblast growth factor-23 regulates parathyroid hormone and 1alphahydroxylase expression in cultured bovine parathyroid cells. J Endocrinol 195: $125-131$.

5. Gutierrez OM, Januzzi JL, Isakova T, Laliberte K, Smith K, et al. (2009) Fibroblast growth factor 23 and left ventricular hypertrophy in chronic kidney disease. Circulation 119: 2545-2552. 
6. Faul C, Amaral AP, Oskouei B, Hu MC, Sloan A, et al. (2011) FGF23 induces left ventricular hypertrophy. J Clin Invest 121: 4393-4408.

7. Mirza MA, Larsson A, Lind L, Larsson TE (2009) Circulating fibroblast growth factor-23 is associated with vascular dysfunction in the community. Atherosclerosis 205: 385-390.

8. Negishi K, Kobayashi M, Ochiai I, Yamazaki Y, Hasegawa H, et al. (2010) Association between fibroblast growth factor 23 and left ventricular hypertrophy in maintenance hemodialysis patients. Comparison with B-type natriuretic peptide and cardiac troponin T. Circ J 74: 2734-2740.

9. Kitagawa M, Sugiyama H, Morinaga H, Inoue T, Takiue K, et al. (2013) A decreased level of serum soluble klotho is an independent biomarker associated with arterial stiffness in patients with chronic kidney disease. PLoS One 8: e56695.

10. Dalal M, Sun K, Cappola AR, Ferrucci L, Crasto C, et al. (2011) Relationship of serum fibroblast growth factor 23 with cardiovascular disease in older community-dwelling women. Eur J Endocrinol 165: 797-803.

11. Larsson T, Nisbeth U, Ljunggren O, Juppner H, Jonsson KB (2003) Circulating concentration of FGF-23 increases as renal function declines in patients with chronic kidney disease, but does not change in response to variation in phosphate intake in healthy volunteers. Kidney Int 64: 2272-2279.

12. Go AS, Chertow GM, Fan D, McCulloch CE, Hsu CY (2004) Chronic kidney disease and the risks of death, cardiovascular events, and hospitalization. N Engl J Med 351: 1296-1305.

13. Razzaque MS (2009) The FGF23-Klotho axis: endocrine regulation of phosphate homeostasis. Nat Rev Endocrinol 5: 611-619.

14. Yamazaki Y, Imura A, Urakawa I, Shimada T, Murakami J, et al, (2010) Establishment of sandwich ELISA for soluble alpha-Klotho measurement: Agedependent change of soluble alpha-Klotho levels in healthy subjects. Biochem Biophys Res Commun 398: 513-518.

15. Imamura A, Okumura K, Ogawa Y, Murakami R, Torigoe M, et al. (2006) Klotho gene polymorphism may be a genetic risk factor for atherosclerotic coronary artery disease but not for vasospastic angina in Japanese. Clin Chim Acta 371: 66-70.

16. Foley RN, Collins AJ, Herzog CA, Ishani A, Kalra PA (2009) Serum phosphorus levels associate with coronary atherosclerosis in young adults. J Am Soc Nephrol 20: $397-404$.

17. Ix JH, De Boer IH, Peralta CA, Adeney KL, Duprez DA, et al. (2009) Serum phosphorus concentrations and arterial stiffness among individuals with normal kidney function to moderate kidney disease in MESA. Clin J Am Soc Nephrol 4: 609-615.

18. Kestenbaum B, Katz R, de Boer I, Hoofnagle A, Sarnak MJ, et al. (2011) Vitamin D, parathyroid hormone, and cardiovascular events among older adults. J Am Coll Cardiol 58: 1433-1441.

19. Lopez FL, Agarwal SK, Grams ME, Loehr LR, Soliman EZ, et al. (2012) Relation of Serum Phosphorus Levels to the Incidence of Atrial Fibrillation (from the Atherosclerosis Risk In Communities [ARIC] Study). Am J Cardiol.

20. Yokoyama K, Imura A, Ohkido I, Maruyama Y, Yamazaki Y, et al. (2012) Serum soluble alpha-klotho in hemodialysis patients. Clin Nephrol 77: 347-351.

21. Ibrahim S, Rashed L (2009) Serum fibroblast growth factor-23 levels in chronic haemodialysis patients. Int Urol Nephrol 41: 163-169.

22. Matsuo S, Imai E, Horio M, Yasuda Y, Tomita K, et al. (2009) Revised equations for estimated GFR from serum creatinine in Japan. Am J Kidney Dis 53: $982-992$.

23. Devereux RB, Reichek N (1977) Echocardiographic determination of left ventricular mass in man. Anatomic validation of the method. Circulation 55: 613-618.

24. Wachtell K, Bella JN, Liebson PR, Gerdts E, Dahlof B, et al. (2000) Impact of different partition values on prevalences of left ventricular hypertrophy and concentric geometry in a large hypertensive population: the LIFE study. Hypertension 35: 6-12.

25. Roman MJ, Pickering TG, Schwartz JE, Pini R, Devereux RB (1996) Relation of arterial structure and function to left ventricular geometric patterns in hypertensive adults. J Am Coll Cardiol 28: 751-756.
26. Isakova T (2012) Fibroblast growth factor 23 and adverse clinical outcomes in chronic kidney disease. Curr Opin Nephrol Hypertens 21: 334-340.

27. Westerberg PA, Tivesten A, Karlsson MK, Mellstrom D, Orwoll E, et al. (2013) Fibroblast growth factor 23, mineral metabolism and mortality among elderly men (Swedish MrOs). BMC Nephrol 14: 85

28. Arnlov J, Carlsson AC, Sundstrom J, Ingelsson E, Larsson A, et al. (2013) Higher fibroblast growth factor-23 increases the risk of all-cause and cardiovascular mortality in the community. Kidney Int 83: 160-166.

29. Mirza MA, Larsson A, Melhus H, Lind L, Larsson TE (2009) Serum intact FGF23 associate with left ventricular mass, hypertrophy and geometry in an elderly population. Atherosclerosis 207: 546-551.

30. Touchberry CD, Green TM, Tchikrizov V, Mannix JE, Mao TF, et al. (2013) FGF23 is a novel regulator of intracellular calcium and cardiac contractility in addition to cardiac hypertrophy. Am J Physiol Endocrinol Metab.

31. Unsal A, Kose Budak S, Koc Y, Basturk T, Sakaci T, et al. (2012) Relationship of fibroblast growth factor 23 with left ventricle mass index and coronary calcificaton in chronic renal disease. Kidney Blood Press Res 36: 55-64.

32. Westerberg PA, Linde T, Wikstrom B, Ljunggren O, Stridsberg M, et al. (2007) Regulation of fibroblast growth factor-23 in chronic kidney disease. Nephrol Dial Transplant 22: 3202-3207.

33. Wolf M (2012) Update on fibroblast growth factor 23 in chronic kidney disease. Kidney Int 82: 737-747.

34. Kim HR, Nam BY, Kim DW, Kang MW, Han JH, et al. (2013) Circulating alpha-Klotho Levels in CKD and Relationship to Progression. Am J Kidney Dis 61: 899-909.

35. Kacso IM, Bondor CI, Kacso G (2012) Soluble serum Klotho in diabetic nephropathy: relationship to VEGF-A. Clin Biochem 45: 1415-1420.

36. Semba RD, Cappola AR, Sun K, Bandinelli S, Dalal M, et al. (2011) Plasma klotho and cardiovascular disease in adults. J Am Geriatr Soc 59: 1596-1601.

37. Xie J, Cha SK, An SW, Kuro OM, Birnbaumer L, et al. (2012) Cardioprotection by Klotho through downregulation of TRPC6 channels in the mouse heart. Nat Commun 3: 1238.

38. Fall T, Shiue I, Bergea af Geijerstam P, Sundstrom J, Arnlov J, et al. (2012) Relations of circulating vitamin $\mathrm{D}$ concentrations with left ventricular geometry and function. Eur J Heart Fail 14: 985-991.

39. van Ballegooijen AJ, Visser M, Kestenbaum B, Siscovick DS, de Boer IH, et al. (2013) Relation of Vitamin D and Parathyroid Hormone to Cardiac Biomarkers and to Left Ventricular Mass (from the Cardiovascular Health Study). Am J Cardiol 111: 418-424.

40. Kamycheva E, Wilsgaard T, Schirmer H, Jorde R (2013) Serum 25hydroxyvitamin $\mathrm{D}$ and left ventricular systolic function in a non-smoking population: the Tromso Study. Eur J Heart Fail.

41. Gosse $\mathrm{P}$ (2005) Left ventricular hypertrophy as a predictor of cardiovascular risk. J Hypertens Suppl 23: S27-33.

42. John GB, Cheng CY, Kuro-o M (2011) Role of Klotho in aging, phosphate metabolism, and CKD. Am J Kidney Dis 58: 127-134.

43. Heine GH, Seiler S, Fliser D (2012) FGF-23: the rise of a novel cardiovascular risk marker in CKD. Nephrol Dial Transplant 27: 3072-3081.

44. Larsson TE, Olauson H, Hagstrom E, Ingelsson E, Arnlov J, et al. (2010) Conjoint effects of serum calcium and phosphate on risk of total, cardiovascular, and noncardiovascular mortality in the community. Arterioscler Thromb Vasc Biol 30: 333-339.

45. Grandi NC, Breitling LP, Brenner H (2010) Vitamin D and cardiovascular disease: systematic review and meta-analysis of prospective studies. Prev Med 51: 228-233.

46. Sugimoto T, Dohi K, Onishi K, Watanabe K, Sato Y, et al. (2013) Interrelationship between haemodynamic state and serum intact parathyroid hormone levels in patients with chronic heart failure. Heart 99: 111-115.

47. Silver J, Naveh-Many T (2010) FGF23 and the parathyroid glands. Pediatr Nephrol 25: 2241-2245.

48. Seiler S, Cremers B, Rebling NM, Hornof F, Jeken J, et al. (2011) The phosphatonin fibroblast growth factor 23 links calcium-phosphate metabolism with left-ventricular dysfunction and atrial fibrillation. Eur Heart J 32: 26882696. 